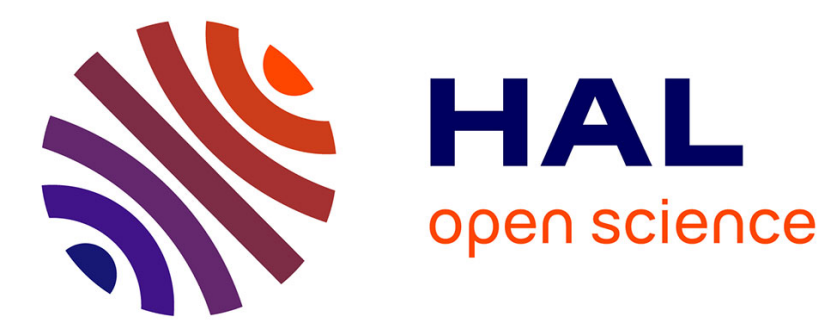

\title{
Sparse + smooth decomposition models for multi-temporal SAR images
}

Sylvain Lobry, Loïc Denis, Florence Tupin

\section{To cite this version:}

Sylvain Lobry, Loïc Denis, Florence Tupin. Sparse + smooth decomposition models for multi-temporal SAR images. 8th International Workshop on the Analysis of Multitemporal Remote Sensing Images (Multi-Temp), Jul 2015, Annecy, France. 10.1109/Multi-Temp.2015.7245772 ujm-01219129

\section{HAL Id: ujm-01219129 \\ https://hal-ujm.archives-ouvertes.fr/ujm-01219129}

Submitted on 22 Oct 2015

HAL is a multi-disciplinary open access archive for the deposit and dissemination of scientific research documents, whether they are published or not. The documents may come from teaching and research institutions in France or abroad, or from public or private research centers.
L'archive ouverte pluridisciplinaire HAL, est destinée au dépôt et à la diffusion de documents scientifiques de niveau recherche, publiés ou non, émanant des établissements d'enseignement et de recherche français ou étrangers, des laboratoires publics ou privés. 


\section{Sparse + smooth decomposition models for multi-temporal SAR images}

\author{
Sylvain Lobry \\ Institut Telecom, Telecom ParisTech \\ CNRS LTCI \\ Paris, France
}

\author{
Loïc Denis \\ Laboratoire Hubert Curien \\ UMR 5516 CNRS \\ Universite de Saint-Etienne \\ and Telecom Saint-Etienne \\ Saint-Etienne, France
}

\author{
Florence Tupin \\ Institut Telecom, Telecom ParisTech \\ CNRS LTCI \\ Paris, France
}

\begin{abstract}
SAR images have distinctive characteristics compared to optical images: speckle phenomenon produces strong fluctuations, and strong scatterers have radar signatures several orders of magnitude larger than others. We propose to use an image decomposition approach to account for these peculiarities.
\end{abstract}

Several methods have been proposed in the field of image processing to decompose an image into components of different nature, such as a geometrical part and a textural part. They are generally stated as an energy minimization problem where specific penalty terms are applied to each component of the sought decomposition.

We decompose temporal series of SAR images into three components: speckle, strong scatterers and background. Our decomposition method is based on a discrete optimization technique by graph-cut. We apply it to change detection tasks.

\section{INTRODUCTION}

Denoising and restoration is one of the oldest challenges of image processing. Like other coherent imaging techniques, SAR imagery suffers from strong fluctuations due to speckle phenomenon. Speckle can be described as a multiplicative noise, while most algorithms developed in the field of image processing are designed to deal with an additive noise. This calls for an adaptation of algorithms developed for optical images or the development of new techniques.

The simplest way to reduce the noise is multi-looking which amounts to averaging pixel values within a small window. With a window big enough, noise can be strongly reduced, at the cost of a resolution loss. This kind of method is not well suited to areas with bright scatterers. Point-like strong scatterers have a high contrast compared to the background and are typically caused by man-made structures, such as buildings. Local averaging methods need to deal with these points separately in order to prevent from spreading them out on the whole window. Strong scatterers can be identified using for instance a likelihood ratio test as in [1] and [2]. The corresponding detector compares the radiometry of the point under test to the average value computed over a surrounding window (e.g., cross-shaped). While being simple, this test fails when other scatterers are present in the window.

This work has been funded by the CNES and the "Future et Ruptures" program.
To cope with these problems related to the specific nature of the SAR images, we propose to model separately these two contributions (background and bright scatterers) and to jointly estimate them. This paper extends the idea of SAR image decomposition introduced in [3] and in [4] to temporal series and applies it to change detection.

This decomposition is then applied to detect changes between SAR images taken at different times. Detecting and analyzing changes is one of the primary application for SAR data, mainly thanks to the reliability of acquisition regarding the weather. The problem has first been tackled by [5] using a difference operator. State-of-the art methods now use likelihood ratio test such as in [6] and in [7].

In section II, we describe a model to detect strong scatterers and perform change detection. This model is stated as a minimization problem. In section III, we provide a way of obtaining the exact solution for this problem. Finally, we describe and evaluate our models in an application of change detection in section IV.

\section{MULTI-TEMPORAL SPARSE + SMOOTH DECOMPOSITION MODEL}

Total variation (TV) minimization [8] is a method widely used for noise reduction. It strongly penalizes signal fluctuations while preserving edges by minimizing the total variation of the signal (i.e., the sum of the spatial gradient magnitude).

Although this method has been applied by several authors to speckle noise reduction in SAR imagery (see [9]-[13]), it is not well adapted to urban areas where punctual bright scatterers are frequent. Indeed, bright scatterers are points with a radiometric value several orders of magnitude higher than the surrounding background. Using total variation regularization for such signals has the effect of removing the point-like scatterers and of biasing the estimation of the neighboring area (spreading effect). On the other hand, these points are sparsely distributed in the image and are thus present in a limited number. In this section, we propose a decomposition model combining regularity of the background and sparsity of the bright scatterers in multi-temporal series.

We consider a temporal series of observed images $\mathbf{V}=$ $\left\{\mathbf{v}_{1}, \ldots, \mathbf{v}_{n}\right\}$ where $\mathbf{v}_{t}$ is the observed image at the $t$-th date. The underlying (noiseless) scene is $\mathbf{U}=\left\{\mathbf{u}_{1}, \ldots, \mathbf{u}_{n}\right\}$. The 
scene $\mathbf{u}_{t}$ at the $t$-th date is modeled as a sum of 2 components: $\mathbf{u}_{\mathrm{BV} t}$ (component with bounded variations, representing the background) and $\mathbf{u}_{\mathbf{S} t}$ (sparse component representing the bright scatterers). The observed image $\mathbf{v}_{t}$ is related to the underlying scene $\mathbf{u}_{t}$ by:

$$
\mathbf{v}_{t}=\left(\mathbf{u}_{\mathbf{B} \mathbf{v}_{t}}+\mathbf{u}_{\mathbf{S} t}\right) \times \mathbf{n}_{t}
$$

where $\mathbf{n}_{t}$ is a speckle noise realization corresponding to a SAR acquisition system with multiplicative noise model. In the case of SAR images, noise follows a Rayleigh distribution (when considering amplitude images) and is weakly correlated (if images are not over-sampled) so that the likelihood distribution can be considered separable:

$$
\begin{aligned}
\mathrm{p}(\mathbf{V} \mid \mathbf{U}) & =\prod_{t=1}^{n} \prod_{i \in \Omega} \mathrm{p}\left(\mathbf{v}_{t}(i) \mid \mathbf{u}_{t}(i)\right) \\
& =\prod_{t=1}^{n} \prod_{i \in \Omega} \frac{2 \mathbf{v}_{t}(i)}{\mathbf{u}_{t}^{2}(i)} \exp \left(\frac{-\mathbf{v}_{t}^{2}(i)}{\mathbf{u}_{t}^{2}(i)}\right),
\end{aligned}
$$

where $\Omega$ is the set of the pixels in an image and $\mathbf{u}_{t}(i)$ is the value at pixel $i$ in image $t$.

We define next the prior $\mathrm{p}(\mathbf{U})$ in order to enforce the decomposition of the radar scene into its two independent components $\mathbf{U}_{\mathbf{B V}}$ and $\mathbf{U}_{\mathbf{S}}$ :

$$
-\log \mathrm{p}(\mathbf{U})=-\beta_{B V} \log \mathrm{p}\left(\mathbf{U}_{\mathbf{B V}}\right)-\beta_{S} \log \mathrm{p}\left(\mathbf{U}_{\mathbf{S}}\right),
$$

where the distributions $\mathrm{p}\left(\mathbf{U}_{\mathbf{B V}}\right)$ and $\mathrm{p}\left(\mathbf{U}_{\mathbf{S}}\right)$ model each component. The background is consider to be formed by homogeneous regions with sharp boundaries (piece wise constant model), with possibly some abrupt changes between two dates. Images following such models have a low 3D total variation, we therefore choose to define:

$$
\begin{aligned}
-\log \mathbf{p}\left(\mathbf{U}_{\mathbf{B V}}\right) & =\mathrm{TV}_{3 \mathrm{D}}\left(\mathbf{U}_{\mathbf{B V}}\right) \\
& =\sum_{t=1}^{n}\left(\sum_{i, j \in \mathcal{C}}\left|\mathbf{u}_{\mathbf{B V} t}(i)-\mathbf{u}_{\mathbf{B V} t}(j)\right|\right) \\
& +\sum_{t=1}^{n-1}\left(\sum_{i \in \Omega}\left|\mathbf{u}_{\mathbf{B V} t}(i)-\mathbf{u}_{\mathbf{B V} t+1}(i)\right|\right),
\end{aligned}
$$

where $\mathcal{C}$ is the set of all cliques in $\Omega$ depending of the chosen neighborhood. The choice of an anisotropic version of total variation (the gradient magnitude is defined in the L1 norm sense) will find its justification in the numerical optimization method described in the next section. The component containing strong scatterers is sparse, which is classically enforced by an energy that increases with the number of non-zero elements, such as the L0 pseudo-norm:

$$
-\log \mathrm{p}\left(\mathbf{U}_{\mathbf{S}}\right)=\left\|\mathbf{U}_{\mathbf{S}}\right\|_{0}
$$

Up to a constant term, the (opposite) log posterior distribution corresponds to the following energy:

$$
\mathcal{E}(\mathbf{U})=-\log (\mathrm{p}(\mathbf{V} \mid \mathbf{U}))-\log (\mathrm{p}(\mathbf{U}))
$$

with:

$$
\begin{aligned}
-\log (\mathbf{p}(\mathbf{V} \mid \mathbf{U})) & =-\log \left(\prod_{t=1}^{n} \prod_{i \in \Omega} \frac{2 \mathbf{v}_{t}(i)}{\mathbf{u}_{t}^{2}(i)} \exp \left(\frac{-\mathbf{v}_{t}^{2}(i)}{\mathbf{u}_{t}^{2}(i)}\right)\right) \\
& =\sum_{t=1}^{n} \sum_{i \in \Omega}\left\{-\log \left(2 \mathbf{v}_{t}(i)\right)\right. \\
& +2 \log \left(\mathbf{u}_{\mathbf{B} \mathbf{V}_{t}}(i)+\mathbf{u}_{\mathbf{S} t}(i)\right) \\
& \left.+\frac{\mathbf{v}_{t}^{2}(i)}{\left(\mathbf{u}_{\mathbf{B} \mathbf{V}_{t}}+\mathbf{u}_{\mathbf{S} t}\right)^{2}(i)}\right\} \\
& =\operatorname{DT}\left(\mathbf{v}_{t}, \mathbf{u}_{\mathbf{B} \mathbf{V} t}, \mathbf{u}_{\mathbf{S} t}\right),
\end{aligned}
$$

with the first term $\left(-\log \left(2 \mathbf{v}_{t}(i)\right)\right)$ independent of $\mathbf{U}$, which will thus be discarded in the following. In summary, the energy is given by:

$$
\begin{aligned}
\mathcal{E}(\mathbf{U}) & =\sum_{t=1}^{n} \beta_{S} \mathrm{LO}\left(\mathbf{u}_{\mathbf{S} t}\right)+\beta_{B V} \mathrm{TV}_{3 \mathrm{D}}\left(\mathbf{U}_{\mathbf{B V}}\right) \\
& +\mathrm{DT}\left(\mathbf{v}_{t}, \mathbf{u}_{\mathbf{B V} t}, \mathbf{u}_{\mathbf{S} t}\right)
\end{aligned}
$$

\section{EXACT DISCRETE OPTIMIZATION BY GRAPH-CUTS}

The cost function introduced in equation 7 is highly nonconvex. In this section, we show how to find the global optimum of the optimization problem (up to a given precision defined by the quantization step size) without resorting to an approximation by $\mathrm{L} 1$ relaxation of the L0-norm, as typically done when solving such optimization problem.

First, we consider the sub-problem of solving $\arg \min _{\mathbf{U}_{S}} \mathcal{E}(\mathbf{U})$ for a fixed $\mathrm{U}_{\mathrm{BV}}$ :

$$
\begin{aligned}
\widehat{\mathbf{U}_{S}}\left(\mathbf{U}_{\mathbf{B V}}\right) & =\arg \min _{\mathbf{U}_{S}} \sum_{t=1}^{n}\left(\mathrm{DT}\left(\mathbf{v}_{t}, \mathbf{u}_{\mathbf{B V} t}, \mathbf{u}_{\mathbf{S} t}\right)+\beta_{S} \mathrm{LO}\left(\mathbf{u}_{\mathbf{S} t}\right)\right) \\
& +\beta_{B V} \mathrm{TV}_{3 \mathrm{D}}\left(\mathbf{U}_{\mathbf{B V}}\right) .
\end{aligned}
$$

Since all terms are separable, we can solve the minimization independently for each pixel:

$\widehat{\mathbf{u}_{\mathbf{S} t}(i)}\left(\mathbf{u}_{\mathbf{B} \mathbf{V}_{t}}(i)\right)= \begin{cases}\mathbf{u}_{\mathbf{S} t}(i)^{\star} & \text { if } \mathrm{DT}\left(\mathbf{v}_{t}(i), \mathbf{u}_{\mathbf{B V} t}(i), \mathbf{u}_{\mathbf{S} t}(i)^{\star}\right) \\ & +\beta_{S}<\mathrm{DT}\left(\mathbf{v}_{t}(i), \mathbf{u}_{\mathbf{B V} t}(i), 0\right) \\ 0 & \text { otherwise }\end{cases}$

With $\mathbf{u}_{\mathbf{S} t}(i)^{\star}=\arg \min _{\mathbf{u}_{\mathbf{S} t}(i)} \mathrm{DT}\left(\mathbf{v}_{t}(i), \mathbf{u}_{\mathbf{B V} t}(i), \mathbf{u}_{\mathbf{S} t}(i)\right)$.

We can now rewrite the minimization problem under a form involving only $\mathbf{U}_{\mathbf{B V}}$ :

$$
\begin{aligned}
\arg \min _{\mathbf{u}_{\mathbf{B V}}} \mathcal{E}(\mathbf{U})= & \arg \min _{\mathbf{u}_{\mathbf{B V}}} \sum_{t=1}^{n} \mathrm{DT}\left(\mathbf{v}_{t}, \mathbf{u}_{\mathbf{B V} t}, \widehat{\mathbf{u}_{\mathbf{S} t}}\left(\mathbf{u}_{\mathbf{B V} t}\right)\right) \\
& +\beta_{S}\left\|\widehat{\mathbf{u}_{\mathbf{S}}}\left(\mathbf{u}_{\mathbf{B V}}\right)\right\|_{0} \\
& +\beta_{B V} \mathrm{TV}_{3 \mathrm{D}}\left(\mathbf{U}_{\mathbf{B V}}\right)
\end{aligned}
$$

The first two terms of equation 8 are separable (i.e., they correspond to a sum of terms involving a single pixel at a time) and the last one is convex and involves only pairwise terms (i.e., pairs of pixel values). This minimization problem can thus be exactly minimized by graph-cuts using the method described in [14]. This optimization method consists

if seen as a discrete optimization problem, i.e., for a given quantization of the component $\mathbf{u}_{\mathrm{BV}}$ 


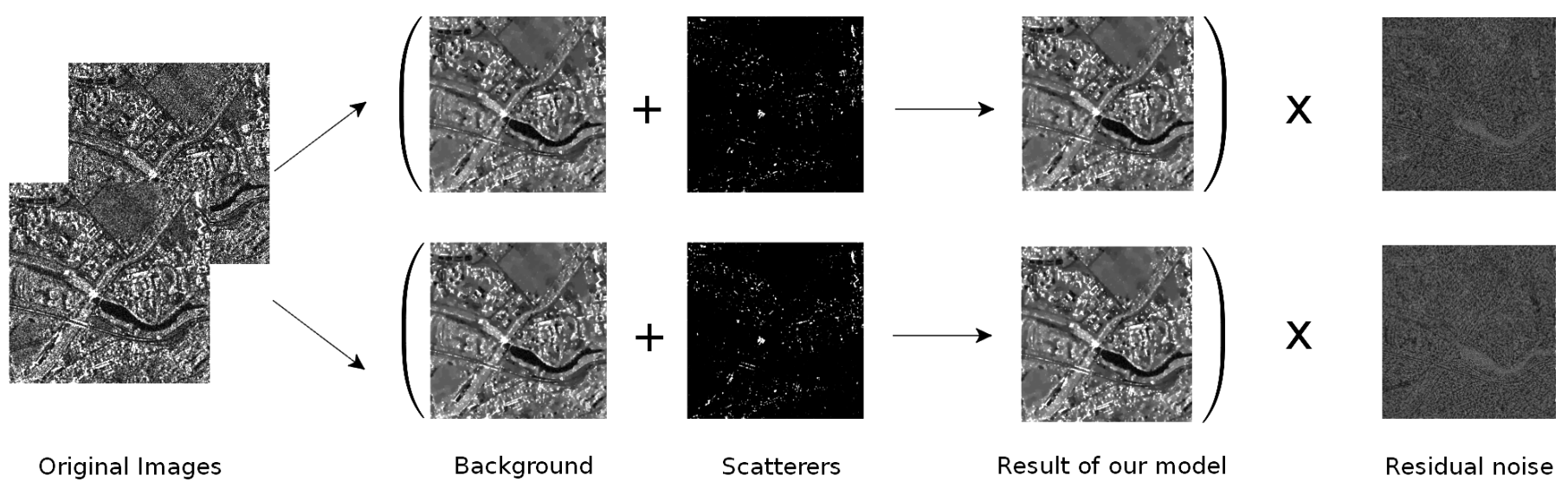

Fig. 1: Decomposition of an image of Saint-Gervais acquired by TerraSAR-X. Thanks to the German Aerospace Agency (DLR) for the images (project MTH0232 and LAN1746).

in searching the min-cut in a graph where every possible value for each pixel is represented by a node. Neighboring nodes (spatially or in time) are interconnected by vertices with weights corresponding to $\beta_{B V}$. Nodes representing the same pixel $i$ at two consecutive possible values $\alpha$ and $\alpha+1$ are interconnected by vertices with weights $\mathrm{p}\left(\mathrm{v}_{t}(i) \mid \alpha\right)$. We show an example of a decomposition obtained with our model using this optimization technique in figure 1 .

Note that the graph construction used here presents a high memory usage: it requires $V=n \times|\Omega| \times|A|$ vertices and $E=7 n \times|\Omega| \times|A|$ edges where $n$ is the number of images in the series, $|\Omega|$ is the number of pixels in each image and $|A|$ the number of possible values for the background image. In the implementation of the min-cut algorithm introduced in [15], a vertex takes 48 bytes and an edge takes 32 bytes. Therefore, the graph construction requires $272 \times n \times|\Omega| \times|E|$ bytes, limiting the size of the images that can be processed. On a larger image, we could use algorithms such as those described in [10] or in [16] that sub-sample the set of amplitudes to lower the memory occupation of the optimization technique and obtain an approximate solution to the problem.

Regarding computational cost, it has a worst-case complexity of $O\left(E V^{2}|C|\right)$ where $|C|$ is the minimum cut and is also the complexity of our method. On a computer with an Intel Xeon(R) CPU E5-1620 with 16Gb of RAM, the algorithm takes 52,04s to compute on 2 images of $300 \times 400$ pixels with 50 levels of quantification. Note that we do not fully benefit from the power of the processor as the implementation of the algorithm is single-core.

\section{SCATTERERS CHANGE DETECTION}

\section{A. Method}

For a series of observed images $\mathbf{V}$, the model described in section II provides a serie of decompositions $\left\{\left(\mathbf{u}_{\mathbf{B V} 1}, \mathbf{u}_{\mathbf{S}_{1} 1}\right), \ldots,\left(\mathbf{u}_{\mathbf{B V} n}, \mathbf{u}_{\mathbf{S}_{n}}\right)\right\}$. A first change detection approach consists of exploiting the scatterer detection to find changes between them. This is not a straightforward task since the value and the exact position of the detected scatterers can greatly vary when a small change happens in the scene. Therefore, we start by binarizing the scatterers images $\left\{\mathbf{u}_{\mathbf{S} t}\right\}$

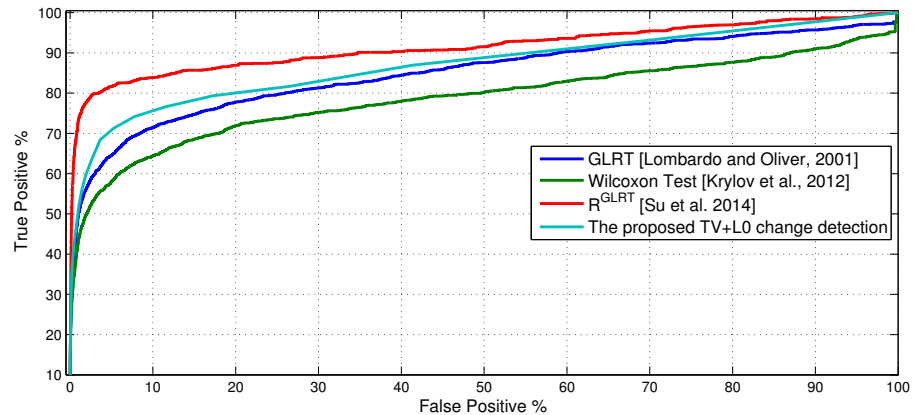

Fig. 2: False positive alarm versus true positive curves of various change detection algorithms

that we name in the following $\left\{\mathbf{u}_{\mathbf{S}_{t}^{\text {bin }}}\right\}$. We describe the procedure for change detection between two images $\mathbf{u}_{1}$ and $\mathbf{u}_{2}$ taken in these series.

The main difficulty to overcome when performing change detection based on point-like scatterers is that these points are not necessarily stable. To handle this situation, we look for pixels that are detected as scatterers in an image without any scatterer detected in a neighborhood in the other image. This can be formalized by:

$$
T(i)=\left|\sum_{\delta} \mathbf{u}_{\mathbf{S}_{1}^{b i n}}(i+\delta)-\sum_{\delta} \mathbf{u}_{\mathbf{S}_{2}^{b i n}}(i+\delta)\right|
$$

where $T$ is a temporary image, and $T(i)$ represents the chances of having a change at position $i . \delta$ is used to iterate over the neighborhood of the pixel $i$. This image is then thresholded to find the changes between the two images.

\section{B. Results}

We ran this algorithm on a time serie of SAR images of Saint-Gervais (France). We compare the results of this algorithm with other change detection methods in figure 2 and we show an example of results in image 3 . While the results are not as good as dedicated state-of-the-art change detection algorithms such as the ones presented in [17] our 


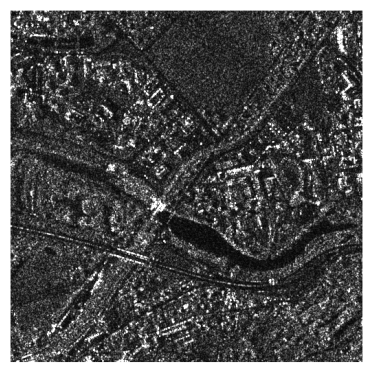

(a) Input image 1 .

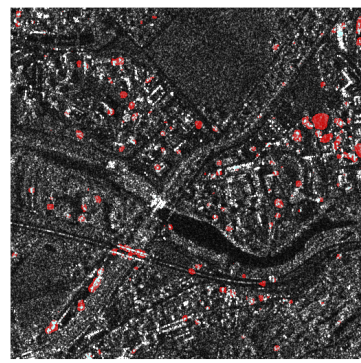

(c) Changes on image 1 .

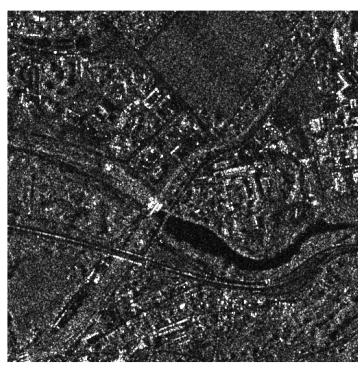

(b) Input image 26 .

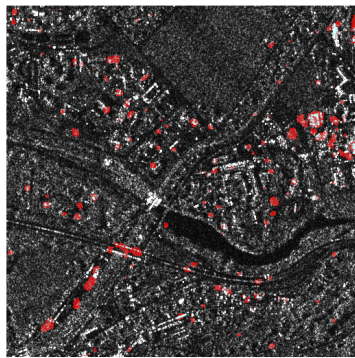

(d) Changes on image 26 .
Fig. 3: Change detection results using the proposed method on images of Saint-Gervais series. Regions with changes that have been detected are indicated in red.

method performs better than the ones presented in [18] and in [19].

\section{CONCLUSION}

This paper introduces a decomposition model suitable to multi-temporal series of SAR images. The proposed model combines a TV regularization with an L0 pseudo-norm to achieve a good estimation of the radiometry of the scene even when point-like bright scatterers are present. It provides a decomposition between the background of the scene and the scatterers. We proposed a simple application of this decomposition framework to change detection using the images of scatterers computed by the algorithm on a SAR series. The method yields results close to that of state-of-the-art algorithms.

Further work includes more complex change detection approaches using all components (background and targets). A contrario framework could be used to detect changes as in [20] from the scatterers images. Furthermore, strategies should be investigated to tackle the practical problem caused by the heavy consumption of memory of our optimization method.

\section{REFERENCES}

[1] A. Lopes, E. Nezry, Goze, R. Touzi, and A. Solaas, "Adaptive processing of multilook complex SAR images," in Geoscience and Remote Sensing Symposium, 1992. IGARSS '92. International, vol. 2, May 1992, pp. 890-892.

[2] A. Lopes, E. Nezry, R. Touzi, and H. Laur, "Structure detection and statistical adaptive speckle filtering in SAR images," International Journal of Remote Sensing, vol. 14, no. 9, pp. 1735-1758, 1993.
[3] J.-F. Aujol, G. Aubert, L. Blanc-Fraud, and A. Chambolle, "Image decomposition application to SAR images," in Scale Space Methods in Computer Vision, ser. Lecture Notes in Computer Science, L. Griffin and M. Lillholm, Eds. Springer Berlin Heidelberg, 2003, vol. 2695, pp. 297-312.

[4] L. Denis, F. Tupin, and X. Rondeau, "Exact discrete minimization for TV+L0 image decomposition models," in Image Processing (ICIP), 2010 17th IEEE International Conference on. IEEE, 2010, pp. 25252528.

[5] A. Singh, "Digital change detection techniques using remotely-sensed data," International journal of remote sensing, vol. 10, no. 6, pp. 9891003, 1989.

[6] X. Su, C.-A. Deledalle, F. Tupin, and H. Sun, "Change detection and classification of multi-temporal SAR series based on generalized likelihood ratio comparing-and-recognizing," in Geoscience and Remote Sensing Symposium (IGARSS), 2014 IEEE International, July 2014, pp. 1433-1436.

[7] G. Quin, B. Pinel-Puyssegur, J.-M. Nicolas, and P. Loreaux, "Mimosa: An automatic change detection method for sar time series," Geoscience and Remote Sensing, IEEE Transactions on, vol. 52, no. 9, pp. 53495363, 2014.

[8] L. I. Rudin, S. Osher, and E. Fatemi, "Nonlinear total variation based noise removal algorithms," Physica D: Nonlinear Phenomena, vol. 60, no. 1 , pp. 259-268, 1992.

[9] G. Aubert and J.-F. Aujol, "A variational approach to removing multiplicative noise," SIAM Journal on Applied Mathematics, vol. 68, no. 4, pp. 925-946, 2008.

[10] L. Denis, F. Tupin, J. Darbon, and M. Sigelle, "SAR image regularization with fast approximate discrete minimization," Image Processing, IEEE Transactions on, vol. 18, no. 7, pp. 1588-1600, July 2009.

[11] G. Steidl and T. Teuber, "Removing multiplicative noise by douglasrachford splitting methods," Journal of Mathematical Imaging and Vision, vol. 36, no. 2, pp. 168-184, 2010.

[12] L. Denis, F. Tupin, J. Darbon, and M. Sigelle, "Joint regularization of phase and amplitude of insar data: Application to 3-d reconstruction," Geoscience and Remote Sensing, IEEE Transactions on, vol. 47, no. 11, pp. 3774-3785, Nov 2009.

[13] F. Palsson, J. R. Sveinsson, M. Ulfarsson, and J. A. Benediktsson, "SAR image denoising using total variation based regularization with SUREbased optimization of the regularization parameter," in Geoscience and Remote Sensing Symposium (IGARSS), 2012 IEEE International, July 2012, pp. 2160-2163.

[14] H. Ishikawa, "Exact optimization for Markov random fields with convex priors," Pattern Analysis and Machine Intelligence, IEEE Transactions on, vol. 25, no. 10, pp. 1333-1336, 2003.

[15] Y. Boykov and V. Kolmogorov, "An experimental comparison of mincut/max-flow algorithms for energy minimization in vision," Pattern Analysis and Machine Intelligence, IEEE Transactions on, vol. 26, no. 9, pp. 1124-1137, 2004.

[16] A. Shabou, F. Tupin, and J. Darbon, "A graph-cut based algorithm for approximate MRF optimization." in ICIP, 2009, pp. 2413-2416.

[17] X. Su, C.-A. Deledalle, F. Tupin, and H. Sun, "NORCAMA: Change Analysis in SAR Time Series by Likelihood Ratio Change Matrix Clustering," ISPRS Journal of Photogrammetry and Remote Sensing, pp. 247-261, May 2014.

[18] P. Lombardo and C. Oliver, "Maximum likelihood approach to the detection of changes between multitemporal SAR images," IEEE Proceedings-Radar, Sonar and Navigation, vol. 148, no. 4, pp. 200210, 2001.

[19] V. Krylov, G. Moser, A. Voisin, B. Serpico, Sebastiano, and J. Zerubia, "Change detection with synthetic aperture radar images by Wilcoxon statistic likelihood ratio test," in IEEE International Conference on Image Processing 2012, Orlando, United States, Sep 2012.

[20] F. Dellinger, J. Delon, Y. Gousseau, J. Michel, and F. Tupin, "Change detection for high resolution satellite images, based on SIFT descriptors and an a contrario approach," in Geoscience and Remote Sensing Symposium (IGARSS), 2014 IEEE International. IEEE, 2014, pp. 1281-1284. 